\title{
FGF23 and primary hyperparathyroidism: is there a link?
}

\author{
Ștefana Catalina Bilha', Alina Andreea Gatu', Cristian Velicescu ${ }^{2}$, Andrei Bilha ${ }^{3}$, Alexandru Florescu', \\ Dumitru Branisteanu ${ }^{1}$
}

${ }^{1}$ Endocrinology Department, "St. Spiridon" Hospital, "Grigore T. Popa" University of Medicine and Pharmacy, Iasi, Romania

${ }^{2}$ Surgery Department, "St. Spiridon" Hospital, "Grigore T. Popa" University of Medicine and Pharmacy, Iasi, Romania

${ }^{3}$ Physiology Department, "Grigore T. Popa" University of Medicine and Pharmacy, Iasi, Romania

\begin{abstract}
Introduction: Data regarding the role of fibroblast growth factor 23 (FGF23) in primary hyperparathyroidism (PHPT) are scarce and discordant. Our study aimed to evaluate the prognostic impact of FGF23 upon the clinical and biochemical evolution of PHPT.

Material and methods: Forty-two patients with ages between 30 and 80 years, diagnosed with PHPT caused by a sporadic, solitary parathyroid adenoma, and referred to surgery (minimally invasive parathyroidectomy) were prospectively included in the study. Serum levels of FGF23, PTH, 25(OH)D3, calcium (Ca), phosphate (P), total procollagen type $1 \mathrm{~N}$-terminal propeptide, and C-terminal telopeptide of type I collagen were determined at baseline (preoperatory), one day after surgery, and in 13 patients also prospectively at three, six, and 12 months. Bone mineral density (BMD) was also evaluated before surgery in all patients and 12 months after surgery in the 13 followed up patients.

Results: In the 42 PHPT patients with D hypovitaminosis (mean 25(OH)D3 levels of $16.2 \pm 1.5 \mathrm{ng} / \mathrm{mL}$ ), preoperatory serum FGF23 concentration was within the normal range $(75.55 \pm 3.39 \mathrm{pg} / \mathrm{mL})$ and remained unchanged one day post operation $(81.69 \pm 4.67 \mathrm{pg} / \mathrm{mL}$, $\mathrm{p}=$ non-significant). The 13 patients followed prospectively for up to 12 months after surgery also showed unmodified FGF23 levels $(80.9 \pm 11.03 \mathrm{pg} / \mathrm{mL}, \mathrm{p}=$ non-significant), despite PTH and Ca normalisation and vitamin D replenishment. Preoperatory FGF23 negatively correlated with PTH $(\mathrm{r}=-0.37, \mathrm{p}=0.038)$, but not with $25(\mathrm{OH}) \mathrm{D} 3, \mathrm{Ca}$, $\mathrm{P}$, bone mass, or metabolism markers.

Conclusions: In PHPT, correlations between FGF23 and PTH seem rather an epiphenomenon. Therefore, we think that FGF23 evaluation and dynamics are not informative regarding PHPT severity. (Endokrynol Pol 2020; 71 (4): 306-312)
\end{abstract}

Key words: FGF23; PTH; vitamin D; parathyroid adenoma; bone

\section{Introduction}

A recently described regulator of calcium (Ca) and phosphate $(\mathrm{P})$ metabolism is osteocyte-synthesised fibroblast growth factor 23 (FGF23) [1]. Through activation of its specific receptor FGFR1 in the presence of klotho, FGF23 downregulates the expression of sodium-P co-transporters, thus inhibiting renal $P$ reabsorption in the proximal renal tubule [2]. It also represses the transcription of the gene that encodes the 1- $\alpha$-hydroxylase, the rate-limiting enzyme for calcitriol synthesis. FGF23 recently emerged as having sodium and chloride conservative functions also in the distal renal tubule, where it increases the expression of epithelial Ca channel TRPV5 and of sodium-chloride cotransporter [1].

The main regulators of $\mathrm{Ca}$ and $\mathrm{P}$ metabolism - parathyroid hormone (PTH), calcitriol and FGF23 - modulate each other's synthesis in conditions of health and disease. Parathyroid hormone (PTH), calcitriol, and P stimulate FGF23 production and secretion, which in turn encompasses a genuine bone-kidney-parathyroid negative feed-back loop by downregulating all the above [2]. However, the FGF23-induced calcitriol downregulation may lead to an indirect elevation of PTH levels [3].

Primary hyperparathyroidism (PHPT) is a disorder characterised by increased serum PTH and calcitriol production - both positive stimuli for FGF23 synthesis - and accompanied by hypophosphataemia and increased urinary $\mathrm{P}$ excretion - modifications also related to FGF23 $[1,2,4,5]$. Despite this, very few studies have approached the secretory profile of FGF23 and its role in the metabolic and bone pathophysiology of PHPT, which remains inconsistently defined [6-9]: apparently, mild elevations of serum FGF23 in PHPT have been reported in some studies before parathyroidectomy with normalisation after surgery [6], but not in others [7]. Furthermore, experimental and also clinical data suggest bone direct autocrine/paracrine actions for FGF23, which is supposed not only to suppress bone formation and mineralisation via Wnt pathway inhibition and downregulation of tissue non-specific 
alkaline phosphatase (TNAP) transcription, but also to act concurrently with PTH upon the expression of the receptor activator of nuclear factor kappa- $\beta$ ligand (RANKL) on osteoblasts and thus promote bone resorption $[1,10,11]$.

Our study aimed at evaluating the prognostic impact of FGF23 upon the clinical, biochemical, and histological evolution and severity of PHPT.

\section{Material and methods}

\section{Study design}

Forty-two patients aged 30-80 years old, diagnosed with PHPT caused by a sporadic, solitary parathyroid adenoma, and referred to surgery (minimally invasive parathyroidectomy) between January and December 2015 were prospectively included in the study after giving their informed consent. The diagnosis of PHPT was established biochemically (increased PTH levels associated with serum Ca above the upper normal reference value of $10 \mathrm{mg} / \mathrm{dL}$ on two subsequent measurements, increased urinary $\mathrm{Ca}$, and creatinine clearance $\geq 90 \mathrm{~mL} / \mathrm{min} / 1.73 \mathrm{~m}^{2}$ ); the parathyroid adenoma was confirmed and localised via parathyroid scintigraphy (Tc99m + MIBI) Exclusion criteria were represented by the conservative treatment of the PHPT, secondary hyperparathyroidism, chronic kidney disease, parathyroid hyperplasia, the presence of multiple endocrine neoplasia and malignant hypercalcaemia.

All 42 patients were evaluated at diagnosis (before parathyroidectomy - serum determinations, bone mineral density), during surgery (parathyroid adenoma volume), and at day 1 post operation (serum determinations), while 13 were followed prospectively at three, six, and 12 months after surgery for the same parameters. None of the patients experienced complications, such as severe hungry bone syndrome, after the parathyroidectomy. Informed consent was obtained from all individual participants included in the study. The study was approved by the local Ethics Committee (reference number 157/6.01.2015).

\section{Serum determinations}

Blood samples were collected at baseline (before surgery) and at day 1 post operation for all patients; blood samples were also collected at three, six, and twelve months for 13 patients that presented for follow-up. For each patient, the serum was aliquoted and stored at $-20^{\circ} \mathrm{C}$ until assessment, which was performed no later than three months after each visit.

Serum and urine concentrations of $\mathrm{Ca}$ and $\mathrm{P}$ were determined via colorimetry using automated analysers (Cobas 6000, Roche). Serum FGF23 (C-terminal; SEA746Hu Wuhan USCN Business Co., Ltd.) and vitamin D levels (25(OH)D3; Immunodiagnostik AG, Bensheim, Germany) were determined via enzyme-linked immunosorbent assay (ELISA), while PTH (Elecsys PTH, Roche Diagnostics GmbH Mannheim, Germany) and bone metabolism markers total procollagen type $1 \mathrm{~N}$-terminal propeptide (P1NP; SEA957Hu Wuhan USCN Business Co., Ltd.) and the C-terminal telopeptide of type I collagen (CTx; CEA892Hu Wuhan USCN Business Co., Ltd.) were measured via electro-chemiluminescence immunoassay (ECLIA).

\section{Intra-operatory adenoma volume}

After surgical excision of the previously localised parathyroid adenoma, the craniocaudal, mediolateral, and anteroposterior diameters were measured, and the volume of the adenoma was calculated as an ellipsoid equation $[12,13]$.

\section{Dual X-ray Absorptiometry (DXA)}

Lumbar spine, hip, and forearm DXA (Hologic Delphi A; Hologic Inc., USA) was performed at baseline (before surgery) for all study participants and at 12 months follow-up for the remaining 13 subjects. Bone mineral density (BMD) was measured by two experienced International Society for Clinical Densitometry certified technicians according to standard protocol. Due to the fact that the $\mathrm{T}$-score was the same as the Z-score in the younger patients (men under 50 years of age and premenopausal women), but not in men over 50 years of age and postmenopausal women, the T-score was employed for all subjects.

\section{Statistical analysis}

Statistical analysis was performed using SPSS 18.0 (SPSS version 18.0 for Windows, IBM SPSS Inc.). Data are expressed as mean \pm standard error of the mean (SEM). Differences between preoperatory and post-operatory values were tested using the paired samples t-test, while ANOVA or the non-parametric Kruskal-Wallis test was employed for differences between three or more variables. Linear relationships between normally distributed variables were investigated with Pearson's correlation coefficient, and non-normally distributed variables with Spearman's correlation coefficient. The chi-squared test was employed to investigate associations between categorical variables. A p value $<0.05$ was considered significant.

\section{Results}

The baseline characteristics of the study participants are shown in Table 1 . The study group had a mean age of $54.6 \pm 1.8$ years and was composed primarily of women. Mean serum FGF23 concentration before surgery was within the normal range $(75.55 \pm 3.39 \mathrm{pg} / \mathrm{mL})$

Table 1. Baseline characteristics of the study participants

\begin{tabular}{lcc}
\hline & $\begin{array}{c}\text { Baseline value } \\
\text { ( } \mathbf{n}=\mathbf{4 2})\end{array}$ & $\begin{array}{c}\text { Reference } \\
\text { range }\end{array}$ \\
\hline Age [years] & $54.6 \pm 1.8$ & \\
\hline Gender (women: men) & $41: 1$ \\
\hline PTH [pg/mL] & $490.4 \pm 189.9$ & $15-65$ \\
\hline Serum total calcium [mg/dL] & $11.9 \pm 0.2$ & $8.4-10.2$ \\
\hline Serum phosphate [mg/dL] & $2.4 \pm 0.1$ & $2.3-4.7$ \\
\hline Urine calcium [mg/24 h] & $318.6 \pm 31.2$ & $<300$ \\
\hline FGF23 [pg/mL] & $75.55 \pm 3.39$ & $<220$ \\
\hline 25(OH)D3 [ng/mL] & $16.2 \pm 1.5$ & $\geq 30$ \\
\hline & & $16,000-96,000$ \\
(women) & $22,000-87,000$ \\
P1NP [pg/mL] & $28648.8 \pm 845.8$ & $($ men) \\
\hline CTx [pg/mL] & & $104-1008$ \\
& $121.5 \pm 20.9$ & (women) \\
\hline Lumbar spine T-score & $-2.45 \pm 0.2$ & $\geq-1$ \\
\hline Femoral neck T-score & $-1.67 \pm 0.2$ & $\geq-1$ \\
\hline 33\% radius T-score & $-2.75 \pm 0.3$ & $\geq-1$ \\
\hline Fragility fractures (yes: no) & $5: 37$ & \\
\hline Adenoma volume [mL] & $3.1 \pm 0.5$ & \\
\hline Co & & 35 (men) \\
\hline
\end{tabular}

Continuous data are expressed as mean \pm standard error of the mean PTH — parathyroid hormone; FGF23 - fibroblast growth factor 23; 25(OH)D3 - vitamin D; P1NP - total procollagen type $1 \mathrm{~N}$-terminal propeptide; CTx — C-terminal telopeptide of type I collagen 


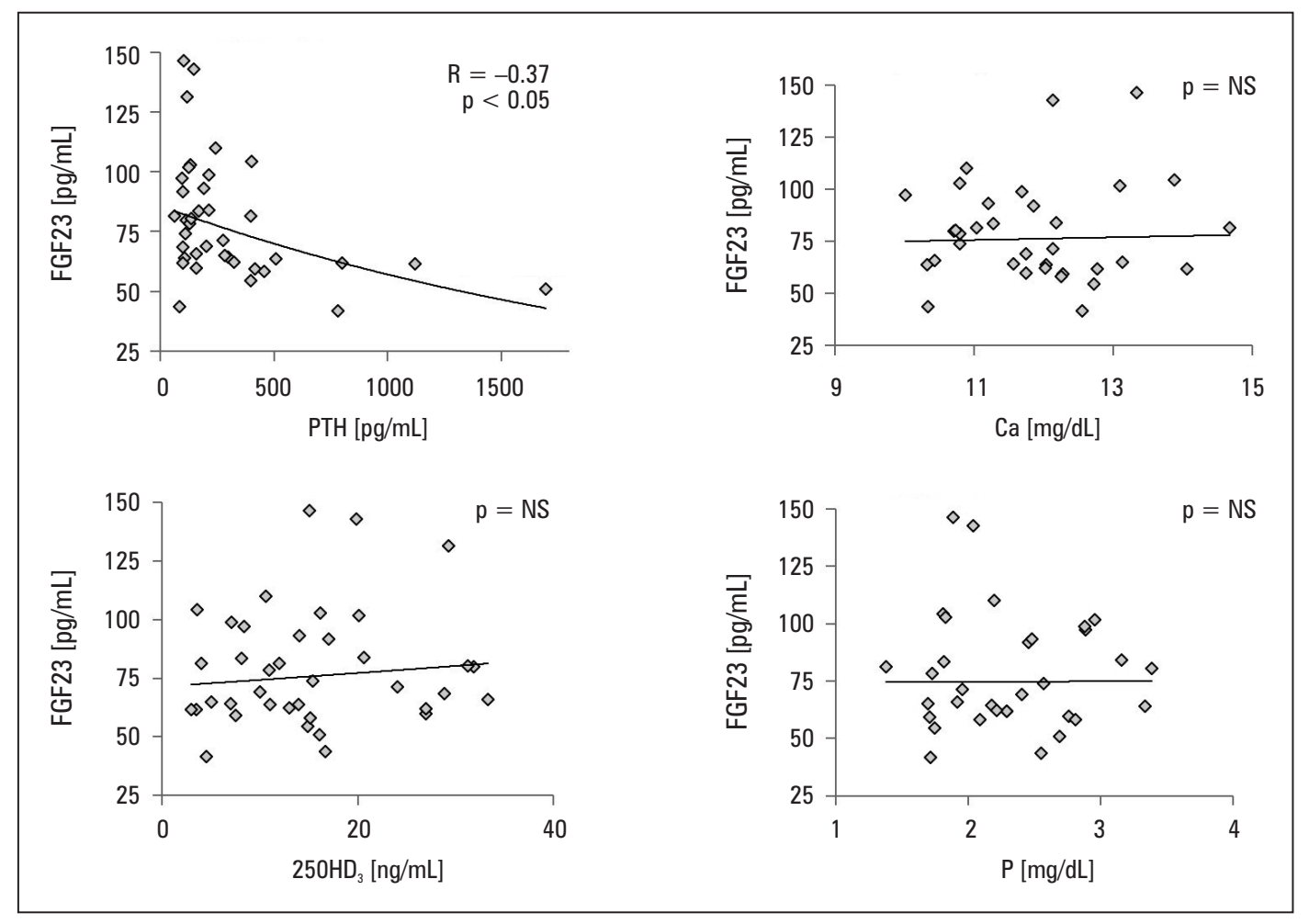

Figure 1. Correlations between fibroblast growth factor 23 (FGF23) and parathormone (PTH), vitamin D (25(OH)D3), calcium, and phosphate in patients with primary hyperparathyroidism (PHPT). Ca — calcium; P — phosphate; PHPT — primary hyperparathyroidism; NS - non-significant; $r$ - correlation coefficient

for patients with PHPT, PTH levels were increased $(490.4 \pm 189.9 \mathrm{pg} / \mathrm{mL})$, and $25(\mathrm{OH}) \mathrm{D} 3$ levels were in the range of deficiency (16.2 $\pm 1.5 \mathrm{ng} / \mathrm{mL}$ ) (Tab. 1).

At baseline (preoperatory), FGF23 was negatively and significantly correlated with PTH $(\mathrm{r}=-0.37$, $\mathrm{p}=0.038)$ but not with $25(\mathrm{OH}) \mathrm{D} 3$, serum $\mathrm{Ca}$, or $\mathrm{P}$ (Fig. 1). Fibroblast growth factor 23 was not significantly related to baseline urine $\mathrm{Ca}$ or $\mathrm{P}$ (data not shown).

Both PTH and FGF23 significantly correlated with the volume of the parathyroid adenoma, but the nature of the relationship was different for each of them $(\mathrm{r}=0.42, \mathrm{p}<0.05$ for PTH and $\mathrm{r}=-0.34, \mathrm{p}<0.05$ for FGF23).

Mean serum PTH decreased to normal values one day after surgery $(32.6 \pm 4.38 \mathrm{pg} / \mathrm{mL}, \mathrm{p}<0.001$ versus preoperatory) in parallel with the normalisation of serum Ca $(9.77 \pm 0.19 \mathrm{mg} / \mathrm{dL}, \mathrm{p}<0.0001$ vs. preoperatory) (Fig. 2). However, mean serum FGF23 levels remained unchanged (81.69 \pm 4.67 $\mathrm{pg} / \mathrm{mL}, \mathrm{p}=$ non-significant) (Fig. 2). Mean serum $\mathrm{P}$ had a tendency to increase immediately after surgery, but the difference did not reach statistical significance $(2.52 \pm 0.11 \mathrm{mg} / \mathrm{dL}, \mathrm{p}=$ non-significant) (Fig. 2). The postoperative change of FGF23 did not correlate with changes in PTH, Ca, or P (data not shown). Also, there were no significant differences regarding baseline biochemical markers and their postoperative changes (PTH, Ca, P, 25[OH]D3) between patients in whom FGF23 increased versus those in whom FGF23 decreased (data not shown). No significant predictors for FGF23 decrease were found. During the one-year follow-up in 13 patients (all under vitamin D3 replenishment), 25(OH)D3 levels normalised (mean 25[OH]D3 serum concentration at 12 months post-operatory of $29.89 \pm 3.84 \mathrm{ng} / \mathrm{mL}, \mathrm{p}<0.05)$, but FGF23 levels remained unchanged 12 months after surgery $(80.9 \pm 11.03 \mathrm{pg} / \mathrm{mL}, \mathrm{p}=$ non-significant $)$ (Fig. 3). Serum PTH completely normalised after one year (Fig. 3).

Almost half of the patients $(n=25)$ had osteoporosis at baseline. FGF23 did not differ significantly between osteoporotic and non-osteoporotics patients $(71.9 \pm 26.3 \mathrm{pg} / \mathrm{mL}$ vs. $75.94 \pm 20.7 \mathrm{pg} / \mathrm{mL}, \mathrm{p}=0.89)$. FGF23 levels were not related to BMD, T-scores at the level of the lumbar spine, hip, and 33\% radius or to CTx and P1NP in the whole group (data not shown) or in the osteoporotics group (Tab. 2). However, FGF23 was negatively correlated with femoral neck T-score (strong correlation) and with P1NP (moderate correlation) in the PHPT non-osteoporosis patients (Tab. 2), but the associations were no longer significant when adjusted for PTH (data not shown). 


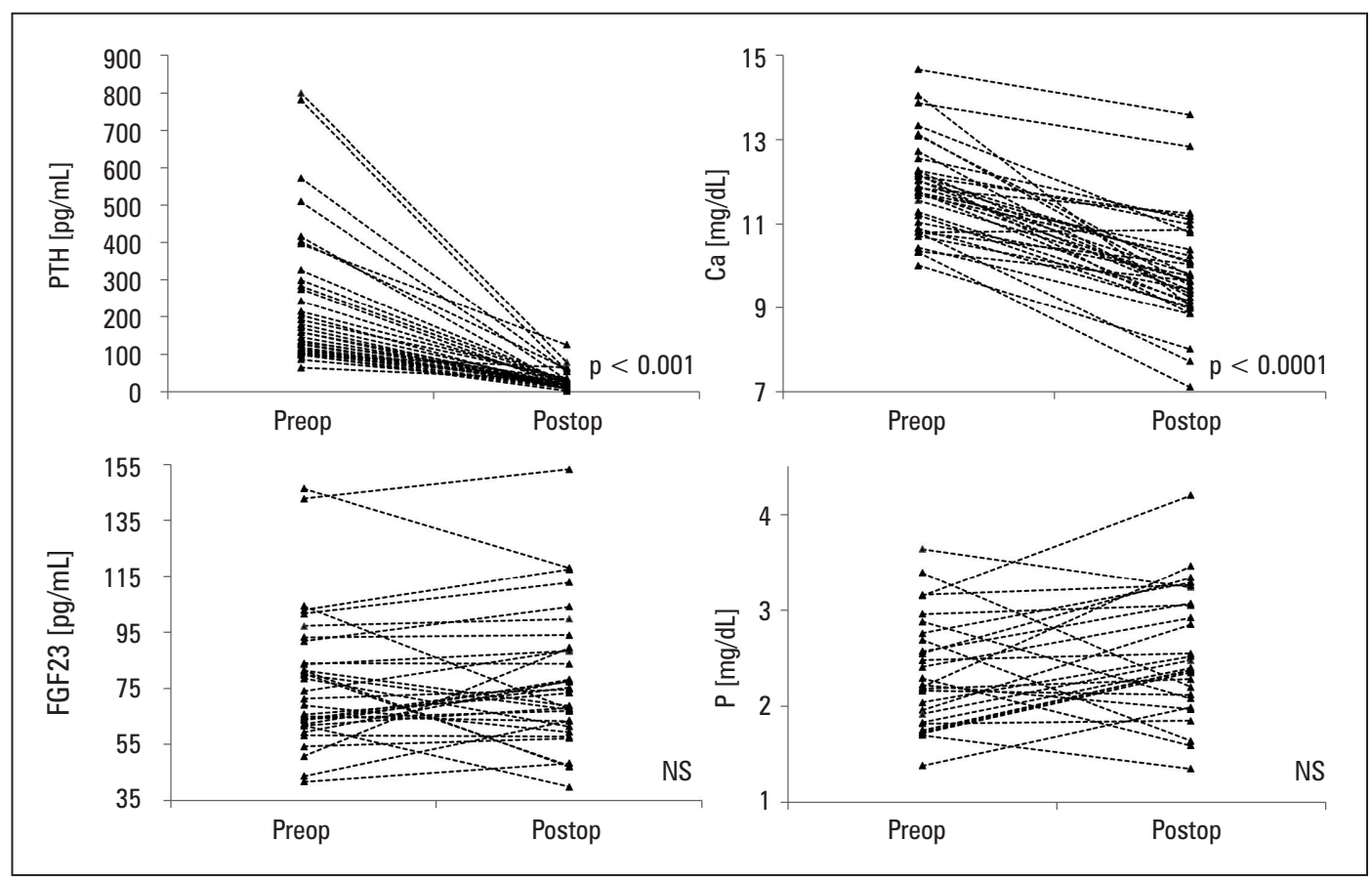

Figure 2. The evolution of the serum parameters after surgery (p-value for pre-versus post-operatory). Ca - calcium; NS — non-significant; P — phosphate; preop — preoperatory; postop — post operation

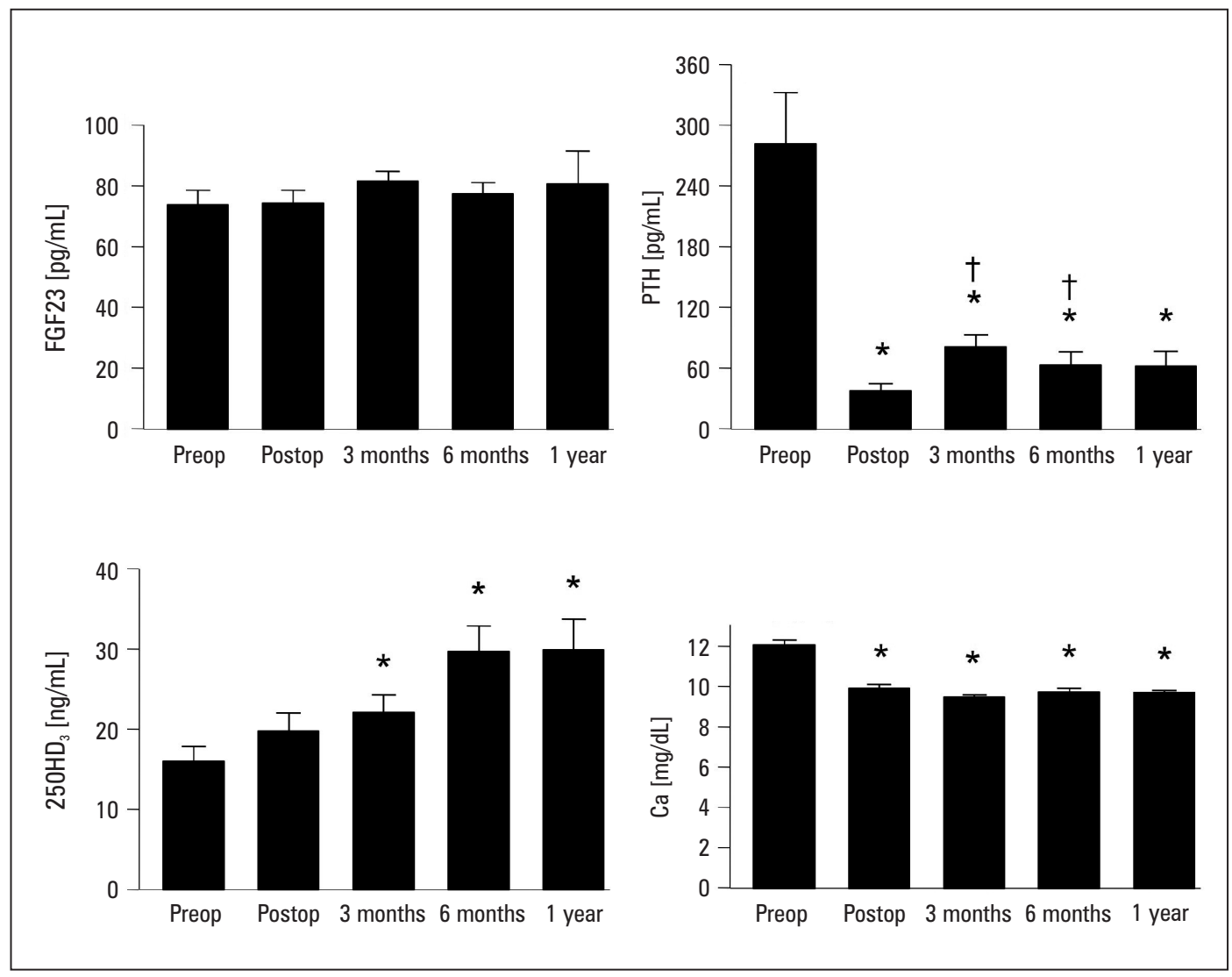

Figure 3. One-year follow-up outcomes in 13 patients (mean \pm standard error of the mean). Ca — calcium; preop - preoperatory; postop — post-operatory; ${ }^{*} p<0.05$ compared to baseline; $t p<0.05$ compared to post operation 
Table 2. Correlation coefficients (followed by p-value) between fibroblast growth factor 23 (FGF23) and calcium and phosphate metabolism parameters and bone mass, respectively, in primary hyperparathyroidism (PHPT) with osteoporosis vs. PHTPT without osteoporosis patients

\begin{tabular}{lcc}
\hline Variable & $\begin{array}{c}\text { Osteoporosis } \\
(\mathbf{n}=\mathbf{2 5}) \\
\text { FGF23 }\end{array}$ & $\begin{array}{c}\text { Without osteoporosis } \\
\text { ( }=\mathbf{~ 1 7 )} \\
\text { FGF23 }\end{array}$ \\
\hline Ca & $-0.11(p=0.68)$ & $-0.24(p=0.4)$ \\
\hline$P$ & $0.1(p=0.63)$ & $0.06(p=0.85)$ \\
\hline PTH & $-0.11(p=0.65)$ & $-0.34(p=0.28)$ \\
\hline 25(OH)D3 & $-0.06(p=0.8)$ & $0.49(p=0.08)$ \\
\hline Lumbar T-score & $-0.37(p=0.13)$ & $0.14(p=0.78)$ \\
\hline Femoral neck T-score & $-0.27(p=0.26)$ & $-\mathbf{0 . 9}(\mathbf{p}=\mathbf{0 . 0 3 7})$ \\
\hline Radius 33\% T-score & $-0.09(p=0.7)$ & $0.32(p=0.48)$ \\
\hline CTx & $0.27(p=0.28)$ & $0.41(p=0.21)$ \\
\hline P1NP & $0.17(p=0.49)$ & $-\mathbf{0 . 6 1}(\mathbf{p}=\mathbf{0 . 0 4 7 )}$ \\
\hline
\end{tabular}

Ca - calcium; $\mathrm{P}$ - phosphate; PTH — parathormone; $25(\mathrm{OH}) \mathrm{D} 3$ - vitamin D; $\mathrm{CTx}$ - C-terminal telopeptide of type I collagen; P1NP — total procollagen type $1 \mathrm{~N}$-terminal propeptide

\section{Discussion}

FGF23 did not vary considerably after the resection of the parathyroid adenoma in PHPT patients with vitamin D deficiency in our study, even after the normalisation of Ca metabolism and despite vitamin D supplementation. FGF23 was not significantly correlated with 25(OH)D3, Ca, and P. Although FGF23 was initially negatively related to bone parameters (femoral neck bone mass and P1NP) in the non-osteoporotic PHPT patients, the associations were lost after adjustment for PTH. The relationships that we identified between FGF23 and PTH or between FGF23 and the volume of the parathyroid adenoma seem rather an epiphenomenon, with the biological action of FGF23 largely taken over by the excessive PTH secretion.

Murine models of PHPT exhibit significantly higher levels of FGF23 compared to wild type mice. In these animal models, FGF23 is positively correlated with serum $\mathrm{Ca}$ and negatively correlated with serum $\mathrm{P}$ at baseline, while significantly decreasing after the surgical excision of the parathyroid glands; this suggests a causal relationship between increased $\mathrm{Ca} / \mathrm{PTH}$ and increased FGF23 levels [14].

However, the few clinical studies addressing FGF23 in PHPT have failed to uniformly demonstrate a significant variation of FGF23 concentrations according to the presence or the cure of PHPT. Despite recording both higher [7, 8] and similar [9] levels of FGF23 in PHPT patients compared to controls and a significant decrease of FGF23 6 hours after surgery [7], initial clinical studies reported no significant changes of FGF23 on day 1, 6, and 7 after parathyroidectomy in PHPT patients with normal renal function [7-9].

More recent clinical data demonstrated a significant six-week postoperative decrease in FGF23 concentrations in 150 PHPT patients who underwent parathyroidectomy; however, besides being vitamin $\mathrm{D}$ replete, patients with abnormal renal function (creatinine clearance down to $40 \mathrm{ml} / \mathrm{min}$ ) were also allowed to enter the study [6]. Although Yamashita et al. [8] also initially found higher FGF23 levels in PHPT compared to normal controls, this was no longer the case when only normal renal function PHPT patients were analysed [8].

Witteveen et al. [4] observed significantly higher FGF23 concentrations in 21 untreated PHPT patients compared to 24 euparathyroid patients cured after successful parathyroidectomy; they were all vitamin D replete, while the studied PHPT group was rather heterogeneous because it also included familial forms of PHPT, such as type 1 multiple endocrine neoplasia, and was compared to a different control group, not to their own evolution after surgery - as we did in the current research. The cross-sectional character of their study also conferred clear interpretative limitations [4].

The higher FGF23 concentrations before surgery compared to different control groups in some studies was explained in the context of PTH-induced hyperproduction of $1,25(\mathrm{OH}) 2 \mathrm{D} 3$, which in turn induces FGF23 expression as a counter-regulatory mechanism for the "danger" of an eventual hyperphosphataemia [15]. FGF23 was, indeed, negatively associated with $1,25(\mathrm{OH}) \mathrm{D} 3$ levels in the study of Witteveen et al. [4]. These authors also found a positive correlation between FGF23 and PTH levels, but only when PHPT patients and surgically cured patients with normal parathyroid function were analysed together; when taken separately, the significant relationship between PTH and FGF23 was not evident anymore [4]. However, they used an intact FGF23 assay, which has a significantly higher intra-individual variation and does not detect the biologically active C-terminal fragment, compared to the C-terminal FGF23 assay [16] that we used in our study.

The entire mechanism through which FGF23 regulates PTH secretion still awaits clarification. A direct (vitamin D-independent) stimulating effect of PTH upon FGF23 has been suggested, especially in conditions associated with excessive PTH production [15, 17]. On the other hand, other lines of evidence demonstrate a klotho-independent FGF23 suppressive effect upon PTH secretion in parathyroid cells [18]. This may partly explain the negative correlation found by us between PTH and FGF23 in our 42 patients with sporadic solitary parathyroid adenomas. 
Primary hyperparathyroidism reflects a new "set point" of the PTH-calcitriol-FGF23-klotho relationship. Walker et al. [19] demonstrated that D hypovitaminosis modifies the biochemical expression spectrum of PHPT [19]. Most of our patients had mild to severe D hypovitaminosis before surgery. However, we did not assess $1,25(\mathrm{OH}) 2 \mathrm{D} 3$ levels in our patients, but it is known that most patients with $\mathrm{D}$ hypovitaminosis exhibit normal levels of $1,25(\mathrm{OH}) 2 \mathrm{D} 3$ in the setting of normal renal function [20]. One may argue that 25(OH)D3 deficiency in our study may have blunted the effect of increased calcitriol levels upon FGF23 and led to "false normal" concentrations of FGF23. Nevertheless, mild D hypovitaminosis does not lead to low $1,25(\mathrm{OH}) 2 \mathrm{D} 3$ [21]. Calcitriol has a serum concentration approximately 1000 -fold less than serum 25(OH)D3; therefore, little substrate is needed to ensure normal circulating levels of $1,25(\mathrm{OH}) 2 \mathrm{D} 3$ [22]. Some papers even found that lower 25-hydroxyvitamin D is linearly associated with higher 1,25-dihydroxyvitamin D levels in PHPT [19]. Moreover, in our study, FGF23 levels remained unchanged after 12 months of follow-up, despite vitamin D supplementation and significant increases in 25(OH)D. Although not assessed in our study, several lines of evidence indicate that klotho expression is down-regulated in parathyroid cells independently of PTH and with no changes in the expression of FGFR1, and it positively correlates with serum $P$ and creatinine $[23,24]$. The role of klotho in the pathophysiology of PHPT remains unclear, especially because not all research [25] found significant klotho expression dysregulation in human parathyroid tissue.

A positive correlation between PTH levels and the volume of the parathyroid adenoma has been described in PHPT [26]. The inverse relationship between FGF23 and the volume of the parathyroid adenoma probably mirrors the FGF23-PTH negative crosstalk rather than a direct effect of FGF23 on the tumour.

Although the FGF23-Ca interplay remains elusive in PHPT patients, the few independent positive associations $[7,8]$ were only reported in the preoperative setting, and FGF23 levels remain fundamentally unchanged after parathyroidectomy, despite normalisation of serum Ca [7-9], including in our study. Furthermore, Nilsson et al. [6] proved that the potential association between FGF23 and serum Ca is weakened by PTH [6]. The parathyroid FGF23 expression is also not related to serum Ca concentration [23]. FGF23 is not independently related to $\mathrm{P}$ in any of the studies [4, 7-9], including ours. It also failed to demonstrate any regulatory effect upon urinary $\mathrm{P}$ in the absence of PTH [17]. It is actually klotho - and not FGF23 - expression level in the parathyroid glands that is associated with serum P [23]. PTH upregulates klotho expression in the renal tubuli, making them responsive to FGF23 and thus highlighting the importance of PTH for renal phosphate elimination [17]. Thus, it is probably only the P retention in the setting of secondary hyperparathyroidism that causes increments in FGF23 production and secretion, as is observed in chronic kidney disease [27].To our knowledge, our study is among the first to investigate noteworthy connections between FGF23 and bone metabolism in PHPT patients: FGF23 is supposed to suppress bone formation via Wnt signalling inhibition, and to stimulate RANKL expression synergic to PTH $[10,11]$. We initially found significant negative correlations between FGF23 and bone mass and metabolism parameters only in PHPT patients without osteoporosis, and not in the whole group, nor in the PHPT patients with osteoporosis. However, the associations were lost when PTH was taken into analysis. A very recent study on PHPT mice demonstrated that the bone transcription of FGF23 is virtually enhanced by PTH via the attenuation of dentin matrix protein 1 (DMP1), a critical element in bone mineralisation [28]. It is, thus, the PTH that has deleterious bone effects, while FGF23 bone production and its correlation with $\mathrm{PTH}$ seem to be merely an epiphenomenon.

The interpretation of our results is limited by the small number of subjects available for follow-up and the absence of a control group. However, we did investigate the hormonal profile of PHPT in a real-life setting of hypovitaminosis D - a prevalent dysfunction in the modern world [29]. Our study is also among the first to investigate the relationship between FGF23 and bone metabolism in PHPT patients.

\section{Conclusions}

FGF23 levels are not significantly changed by autonomous PTH hypersecretion in patients associating $\mathrm{D}$ hypovitaminosis and are not influenced by the surgical excision of the adenoma. The importance of FGF23 in the regulation of $\mathrm{Ca}$ and $\mathrm{P}$ metabolism may be eluded by excessive PTH secretion in the presence of low vitamin D levels. We think that, contrary to secondary hyperparathyroidism, FGF23 measurement cannot be considered a criterion in assessing the severity of PHPT.

\section{Conflict of interest}

The authors declare no conflict of interest.

\section{Funding}

This work was supported through the European Social Fund, Human Resources Development Operational Programme 2007-2013, project no. POSDRU/159/1.5/S/136893. 


\section{References}

1. Erben RG. Physiological Actions of Fibroblast Growth Factor-23. Front Endocrinol (Lausanne). 2018; 9: 267, doi: 10.3389/fendo.2018.00267, indexed in Pubmed: 29892265.

2. Richter B, Faul C. FGF23 Actions on Target Tissues-With and Without Klotho. Front Endocrinol (Lausanne). 2018; 9: 189, doi: 10.3389/fendo.2018.00189, indexed in Pubmed: 29770125.

3. Martin A, David V, Quarles LD. Regulation and function of the FGF23/klotho endocrine pathways. Physiol Rev. 2012; 92(1): 131-155, doi: 10.1152/physrev.00002.2011, indexed in Pubmed: 22298654.

4. Witteveen JE, van Lierop AH, Papapoulos SE, et al. Increased circulating levels of FGF23: an adaptive response in primary hyperparathyroidism? Eur J Endocrinol. 2012; 166(1): 55-60, doi: 10.1530/EJE-11-0523, indexed in Pubmed: 21984611.

5. Grabner A, Mazzaferro S, Cianciolo G, et al. Fibroblast Growth Factor 23: Mineral Metabolism and Beyond. Contrib Nephrol. 2017; 190: 83-95, doi: 10.1159/000468952, indexed in Pubmed: 28535521.

6. Nilsson IL, Norenstedt S, Granath F, et al. FGF23, metabolic risk factors, and blood pressure in patients with primary hyperparathyroidism undergoing parathyroid adenomectomy. Surgery. 2016; 159(1): 211-217, doi: 10.1016/j.surg.2015.06.057, indexed in Pubmed: 26435425.

7. Kobayashi K, Imanishi Y, Miyauchi A, et al. Regulation of plasma fibroblast growth factor 23 by calcium in primary hyperparathyroidism. Eur J Endocrinol. 2006; 154(1): 93-99, doi: 10.1530/eje.1.02053, indexed in Pubmed: 16381997.

8. Yamashita H, Yamashita T, Miyamoto M, et al. Fibroblast growth factor (FGF)-23 in patients with primary hyperparathyroidism. Eur J Endocrinol. 2004; 151(1): 55-60, doi: 10.1530/eje.0.1510055, indexed in Pubmed: 15248822.

9. Tebben PJ, Singh RJ, Clarke BL, et al. Fibroblast growth factor 23, parathyroid hormone, and 1alpha,25-dihydroxyvitamin D in surgically treated primary hyperparathyroidism. Mayo Clin Proc. 2004; 79(12): 1508-1513, doi: 10.4065/79.12.1508, indexed in Pubmed: 15595334.

10. Andrukhova O, Streicher C, Zeitz U, et al. Fgf23 and parathyroid hormone signaling interact in kidney and bone. Mol Cell Endocrinol. 2016; 436: 224-239, doi: 10.1016/j.mce.2016.07.035, indexed in Pubmed: 27498418.

11. Carrillo-López N, Panizo S, Alonso-Montes C, et al. Direct inhibition of osteoblastic Wnt pathway by fibroblast growth factor 23 contributes to bone loss in chronic kidney disease. Kidney Int. 2016; 90(1): 77-89, doi: 10.1016/j.kint.2016.01.024, indexed in Pubmed: 27165819.

12. Zimmermann P, Takala T, Pöyhönen L, et al. Ultrasonography of the thyroid gland in pregnancies complicated by autoimmune thyroid disease. J Clin Ultrasound. 1993; 21(2): 109-113, doi: 10.1002/jcu.1870210206, indexed in Pubmed: 8381129.

13. You SH, Suh SI, Ryoo IS, et al. Assessing the accuracy and reliability of ultrasonographic three-dimensional parathyroid volume measurement in a patient with secondary hyperparathyroidism: a comparison with the two-dimensional conventional method. Ultrasonography. 2017; 36(1): 17-24, doi: 10.14366/usg.16022, indexed in Pubmed: 27457337.

14. Kawata T, Imanishi $Y$, Kobayashi $\mathrm{K}$, et al. Parathyroid hormone regulates fibroblast growth factor- 23 in a mouse model of primary hyperparathyroidism. J Am Soc Nephrol. 2007; 18(10): 2683-2688, doi: 10.1681/ASN.2006070783, indexed in Pubmed: 17855636
15. Erben RG. Update on FGF23 and Klotho signaling. Mol Cell Endocrinol. 2016; 432: 56-65, doi: 10.1016/j.mce.2016.05.008, indexed in Pubmed: 27178987.

16. Smith ER, Cai MM, McMahon LP, et al. Biological variability of plasma intact and C-terminal FGF23 measurements. J Clin Endocrinol Metab. 2012; 97(9): 3357-3365, doi: 10.1210/jc.2012-1811, indexed in Pubmed: 22689697.

17. López I, Rodríguez-Ortiz ME, Almadén Y, et al. Direct and indirect effects of parathyroid hormone on circulating levels of fibroblast growth factor 23 in vivo. Kidney Int. 2011; 80(5): 475-482, doi: 10.1038/ki.2011.107, indexed in Pubmed: 21525854.

18. Olauson H, Lindberg K, Amin R, et al. Parathyroid-specific deletion of Klotho unravels a novel calcineurin-dependent FGF23 signaling pathway that regulates PTH secretion. PLoS Genet. 2013; 9(12): e1003975, doi: 10.1371/journal.pgen.1003975, indexed in Pubmed: 24348262.

19. Walker MD, Bilezikian JP. Vitamin D and primary hyperparathyroidism: more insights into a complex relationship. Endocrine. 2017; 55(1): 3-5, doi: 10.1007/s12020-016-1169-1, indexed in Pubmed: 27858283.

20. Branisteanu D. Sunlight Exposure and Vitamin D Supplementation at the Institutionalized Elderly - Effects on Calcium and Bone Metabolism. Acta Endocrinol (Bucharest). 2007; 3(2): 169-178, doi: 10.4183/aeb.2007.169, indexed in Pubmed: 18088161.

21. Kennel KA, Drake MT, Hurley DL. Vitamin D deficiency in adults: when to test and how to treat. Mayo Clin Proc. 2010; 85(8): 752-7; quiz 757, doi: 10.4065/mcp.2010.0138, indexed in Pubmed: 20675513.

22. de Jongh RT, van Schoor NM, Lips P. Changes in vitamin D endocrinology during aging in adults. Mol Cell Endocrinol. 2017; 453: 144-150, doi: 10.1016/j.mce.2017.06.005, indexed in Pubmed: 28602863.

23. Latus J, Lehmann R, Roesel M, et al. Involvement of -klotho, fibroblast growth factor-, vitamin-D- and calcium-sensing receptor in 53 patients with primary hyperparathyroidism. Endocrine. 2013; 44(1): 255-263, doi: 10.1007/s12020-013-9881-6, indexed in Pubmed: 23334987.

24. Björklund P, Krajisnik T, Akerström G, et al. Type I membrane klotho expression is decreased and inversely correlated to serum calcium in primary hyperparathyroidism. J Clin Endocrinol Metab. 2008; 93(10): 4152-4157, doi: 10.1210/jc.2008-0564, indexed in Pubmed: 18682507.

25. Nakajima K, Okazaki T, Okamoto T, et al. Genes up- or down-regulated by high calcium medium in parathyroid tissue explants from patients with primary hyperparathyroidism. Endocrine Journal. 2009; 57(2): 153-159, doi: 10.1507/endocrj.k09e-321.

26. Gatu A, Velicescu C, Grigorovici A, et al. . The volume of solitary parathyroid adenoma is related to preoperative pth and $250 \mathrm{~h}-\mathrm{d} 3$, but not to calcium levels. Acta Endocrinol (Buchar). 2017; 13(4): 441-446, doi: 10.4183/aeb.2017.441, indexed in Pubmed: 31149214.

27. Silver J, Rodriguez M, Slatopolsky E. FGF23 and PTH - double agents at the heart of CKD. Nephrol Dial Transplant. 2012; 27(5): 1715-1720, doi: 10.1093/ndt/gfs050, indexed in Pubmed: 22447519.

28. Nagata $\mathrm{Y}$, Imanishi $\mathrm{Y}$, Ohara $\mathrm{M}$, et al. Attenuated Dentin Matrix Protein 1 Enhances Fibroblast Growth Factor 23 in Calvaria in a Primary Hyperparathyroidism Model. Endocrinology. 2019; 160(5): 1348-1358, doi: 10.1210/en.2019-00017, indexed in Pubmed: 30916761.

29. Podd D. Hypovitaminosis D: A common deficiency with pervasive consequences. JAAPA. 2015; 28(2): 20-26, doi: 10.1097/01. jaa.0000459810.95512.14, indexed in Pubmed: 25594293. 